\title{
LENGTHENING TEMPORALIS MYOPLASTY AND REDUCTION OF THE SWALLOWING ORAL PHASE DYSFUNCTION IN FACIAL PALSY PATIENTS
}

Benjamin Croiséa,b; Arnaud Paréa, ${ }^{a, b}$ Franck Marmouset ${ }^{c}$; Paul Bregeaut ${ }^{d}$; Aline Jolye; Boris Laure ${ }^{\mathrm{a}, \mathrm{b}, \mathrm{e}}$

${ }^{a}$ Department of Maxillofacial and Plastic Facial Surgery, Trousseau Hospital, 37000 Tours, France

${ }^{b}$ University of François Rabelais, school of medicine, 37000 Tours, France

${ }^{\circ}$ Department of Otolaryngology Head and Neck Surgery, Trousseau Hospital, 37000 Tours, France

${ }^{d}$ Department of Public Health, Bretonneau Hospital, 37000 Tours, France $\square$

e Department of Pediatric Maxillofacial Surgery, Clocheville Hospital, 37000 Tours, France

\section{Corresponding author :}

Benjamin CROISE

Maxillo Facial surgery

CHU Trousseau

Avenvue de la république

37170 Chambray les tours

FRANCE

Email : benjamin.croise.med@gmail.com

Tel +33678387110 


\section{ABSTRACT}

Introduction: Facial palsy can cause dysfunction in the oral phase of swallowing. Lengthening temporalis myoplasty is a widely used technique for correction of facial asymmetry in facial palsy. The aim of this study was to determine whether lengthening temporalis myoplasty could reduce the dysfunction in the oral phase of swallowing in patients with facial palsy.

Materials and Methods: This prospective study enrolled 13 patients undergoing lengthening temporalis myoplasty. Lip continence, bolus residue, and perceived disability before surgery and at 3 months and 6 months after surgery were compared. Lip force was evaluated with a manometric test and drooling with a self-administered questionnaire. Bolus residue was assessed visually. Perceived disability was evaluated using a self-administered questionnaire.

Results: Lip force improved significantly (from $58.23 \pm 23.35 \mathrm{mmHg}$ to $91.15 \pm 18.36$ $\mathrm{mmHg} ; \mathrm{p}=0.001)$. Drooling showed a corresponding reduction, with the score decreasing from $4.31 \pm 1.8$ to $3 \pm 1.41 ; p=0.025$. Decrease in bolus residue was also noted; the score decreased from $1.39 \pm 0.77$ to $0.46 \pm 0.66 ; p<0.001$. These changes contributed to a significant reduction in perceived physical disability; the score decreased from $6.15 \pm 3.74$ to $3.46 \pm 5.70 ; p=0.004)$.

Conclusion: Lengthening temporalis myoplasty, in addition to providing smile reanimation, may also reduce the dysfunction of the oral phase of swallowing in patients with facial palsy.

KEYWORDS: Lengthening temporalis myoplasty, facial paralysis, dysphagia, swallowing dysfunction 
Authors adhere to the STROBE guidelines and the study has been registered on ClinicalTrials.gov under $\mathrm{N}^{\circ} \mathrm{CT} 03284125$.

\section{Introduction}

Facial palsy (FP), which may be due to various causes, has an incidence of 50 per 100000 people per year $(1,2)$. Paralysis of the facial muscles can have major impact on esthetics and function. The facial nerve innervates the buccinator, orbicularis oris, risorius, and zygomatic muscles, all of which have important roles in the oral phase of swallowing (2-5). Many studies have confirmed that FP patients experience swallowing problems. De Swart et al.(6) found that patients with peripheral facial nerve palsy develop eating and drinking problems immediately after disease onset. Seçil et al. (7) reported that $79 \%$ of FP patients have difficulties in managing food in the mouth and 55\% have swallowing dysfunction on electrophysiological analysis. Moverare et al. (8) also reported swallowing problems in $48 \%$ of their patients. Both De Swart et al. (6) and Moverare et al. (8) found that the discomfort level during eating is not linked to the FP severity.

Most patients recover from FP. Facial nerve palsy persisting for $\geq 18$ months is considered permanent $(2,9)$. Various surgical techniques have been used to restore the lower face in patients with permanent FP (10). Currently, one of the most frequently procedure is the lengthening temporalis myoplasty (LTM) that was first described by Labbé (11), even if the gold standard remains the neurotized gracilis (12). LTM has undergone many modifications (13) since its introduction and has proved its efficiency in esthetic restoration and lips reanimation (9). 
The aim of this study was to determine whether LTM could improve the oral phase of swallowing in patients with FP. In order to answer this question, we have proceeded as follows: we quantified the swallowing disorder by measuring lip force, drooling, bolus residue, and perceived disability. The total follow-up was 6 months, with intermediate checkpoints of swallowing at 3 and 6 months.

\section{Materials and Methods}

\subsection{Patients}

This prospective single-institution study was carried out at the Maxillofacial Surgery Department of Tours University Hospital, France, between September 2017 and June 2018. The inclusion criteria were all the patients over 6 years old who had been operated using LTM and who had permanent facial palsy with a Freyss test measuring severity less than 15 . As to the exclusion criteria, all patients with potential impact on swallowing such as TMJ disorder or psychiatric diseases were excluded from our study. Finally, 13 patients were included in our study, these included 12 patients with definitive FP for $>18$ months and one patient with FP due to facial nerve sectioning, with no prospect of short-term recovery. The etiologies included neurinoma of cranial nerve VIII $(n=4)$, meningioma of the cerebellopontine angle $(n$ $=1)$, congenital FP $(n=3)$, Bell palsy $(n=1)$, parotidectomy $(n=2)$, shingles $(n=1)$, and cavernoma bleeding $(n=1)$. All enrolled patients underwent preoperative electromyogram of the facial and temporalis muscles to confirm the diagnosis of FP and to ascertain normal functioning of the temporalis muscle. 
Informed consent was obtained from each patient before inclusion in the study. The regional Ethical Review Board in Caen, France (RIPH3-RNI17/MATPF) approved the study protocol.

\subsection{Surgery}

All 13 patients underwent LTM by the Labbé technique (9); the same surgeon performed all operations. The temporalis muscle was entirely extracted from its fossa, and then osteotomy of the zygomatic arch was performed. The temporalis muscle tendon was spread out along the length of the nasolabial fold, trying to respect the preoperative smile analysis. To avoid elevation of the lip commissure at rest, the temporalis muscle was reintegrated without stress in the temporal fossa. After these procedures the muscle operates as a cutaneous muscle. A coronal approach was adopted for LTM because it allows access to the corrugator and contralateral frontalis muscle to perform myectomies and facilitates ipsilateral scalp resection to lift the eyebrow. No patient received botulinum toxin during the study period.

\subsection{Physiotherapy}

After surgery all patients underwent intensive postoperative reeducation under the supervision of a physiotherapist with specialization in orofacial rehabilitation. Physiotherapy started on the fourth postoperative day with measures to improve lymphatic drainage and limit postoperative edema. From the 21st postoperative day onward, physiotherapy focused on a softening massage of the nasolabial fold scar and the retraining of the temporalis muscle to develop a natural smile. The Maxillofacial Surgery Department protocol includes 3 steps. 
The first one is the temporal mandibular smile ; i.e. the patients have to clench their teeth in front of a mirror in order to smile. The exercises must not be painful (short

and smoothly). The patients are also asked to carry out the exercises at home, 5 to 6 times a day. This step lasts approximately 2 months, once a week with a physiotherapist.

The second step is the voluntary temporal smile. The patients must think of contracting the temporal muscle without clenching their teeth in order to smile. Exercises of muscular relaxation and symmetric and asymmetric smile contraction are also carried out, twice a week with a physiotherapist, and 5 self exercises per day. This step lasts approximately 2 months.

The last step is the spontaneous smile (Fig 1). The patients must not think in order to smile. They work role-playing with the physiotherapist (reading aloud, whistle). The facial expressions are made on order, in imitation or in situation. The patient works with the physiotherapist once every 3 weeks.

No special re-education for swallowing was provided.

\subsection{Evaluation of swallowing}

The oral phase of swallowing extends from the moment the food passes into the mouth till it enters the pharynx. It is a voluntary phase. Patients were evaluated by self-administered questionnaires and clinical tests (performed by an otolaryngologist specialized in swallowing problems). The patients were followed during 6 months after surgery with evaluations carried out at three checkpoints: before surgery, at 3 months after surgery, and at 6 months after surgery. 
Two key aspects of the oral phase of swallowing were evaluated: lip continence and mastication efficiency. Lip continence was evaluated through a clinical test. There is no gold- standard test for assessing lip continence (14). In our study, the patients were asked to contract lips forcefully on a breathing tube balloon linked to a manometer to measure the lip force (in $\mathrm{mmHg}$ ) (Fig 2). Lip continence was also assessed by means of a self-administered questionnaire- - the Drooling Severity and Frequency Scale (DSFS) (15), which is used in Parkinson disease. With this questionnaire drooling severity can be graded on a scale of 1-5 $(1=$ never, $2=$ mild, $3=$ moderate, $4=$ severe, $5=$ profuse $)$ and drooling frequency on a scale of 1-4 $(1=$ never, 2 = occasionally, $3=$ frequently, $4=$ constantly). The total drooling score is the sum of the severity and frequency subscores.

Mastication efficiency was evaluated by having the patient chew a standardized cracker on the paretic side. The mastication time was recorded and the bolus residue at the end of mastication was assessed visually. On this test the score can range from 0-4 $(0=$ no residue, $1=$ residue $<1 / 4,2=$ residue between $1 / 4$ and $1 / 2,3=$ residue between $1 / 2$ and $3 / 4,4=$ residue $>3 / 4$ ).

Disability was evaluated by the self-administered Deglutition Handicap Index (DHI) questionnaire, which includes 30 questions for evaluating three aspects of disability (physical, functional, and emotional/social). Each question is scored on a scale of 0-4 $(0=$ never, $1=$ almost never, $2=$ sometimes, $3=$ almost always, $4=$ always $)$. The maximum possible score is 120 . The handicap is considered slight if the score is $<30$, moderate if the score is $31-60$, and severe if the score is $>61$. 


\subsection{Statistical analysis}

For each test, the mean and the standard deviation were calculated. The measurements were compared using the Wilcoxon test for paired series (Wilcoxon signed-rank test). Data were analyzed with R software (http://www.R-project.org/); $\alpha$ error of $5 \%$ was considered acceptable.

\section{Results}

\subsection{Demographic data}

A total of 13 patients ( 7 female, 6 male) with a mean age of 49 years (range, 9-80 years) were enrolled in this study (table 1). One of the patients did not attend the follow-up at 3 months after surgery; nevertheless, we included the patient in the analysis.

\subsection{Lip continence}

Lip continence showed steady improvement with time after surgery. The manometric scores were $58.23 \pm 23.35 \mathrm{mmHg}$ before surgery vs. $74.33 \pm 20.92 \mathrm{mmHg}$ at 3 months after surgery vs. $91.15 \pm 18.36 \mathrm{mmHg}$ at 6 months after surgery (Fig 3 ). The differences from baseline were statistically significant at both time points after surgery $(p=0.011$ at 3 months and $p=0.001$ at 6 months).

\subsection{Drooling}

Drooling showed steady decrease after surgery. The mean DSFS was $4.31 \pm 1.8$ before surgery vs. $3.83 \pm 1.85$ at 3 months after surgery vs. $3.0 \pm 1.41$ at 6 months 
after surgery (Fig 4). The difference from baseline was not significant at 3 months ( $p$ $=0.275)$; however, at 6 months the difference was statistically significant $(p=0.025)$.

\subsection{Mastication}

The bolus residue at the end of mastication showed a steady decrease over time. The mean score was $1.39 \pm 0.77$ before surgery vs. $0.75 \pm 0.75$ at 3 months after surgery vs. $0.46 \pm 0.66$ at 6 months after surgery (Fig 5). The difference from baseline value was statistically significant at 3 months and at 6 months $(p=0.003$ and $\mathrm{p}<0.001)$.

The mean mastication duration was $3.52 \pm 1.52$ minutes before surgery vs. $3.28 \pm$ 1.37 minutes at 3 months after surgery vs. $3.30 \pm 1.50$ minutes at 6 months after surgery. The changes in mastication duration were not statistically significant difference ( $p=0.10$ at 3 months and $p=0.12$ at 6 months)

\subsection{Disability}

The overall mean DHI score was $12.31 \pm 9.51$ before surgery. The scores for the

individual components were as follows: $6.15 \pm 3.74$ for physical disability; $2.62 \pm 3.57$ for functional disability; and $3.54 \pm 4.79$ for social handicap.

At 3 months after surgery the mean overall DHI score was $9 \pm 10.85$. The score for the individual components of disability were as follows: $4.08 \pm 5.21$ for physical disability; $1.59 \pm 2.43$ for functional disability; and $3.33 \pm 5.99$ for social disability. 
At 6 months after surgery the mean overall DHI score was $8.69 \pm 16.35$. The scores for the individual components were as follows: $3.46 \pm 5.70$ for physical disability; 1.92 \pm 4.41 for functional disability; and $3.31 \pm 6.68$ points for social disability The changes in total DHI score from baseline values were not significant at 3 months or at 6 months after surgery $(p=0.098$ and $p=0.104$, respectively). However, the change in the physical handicap score was significant at both time points $(p=0.032$ at 3 months and $p=0.004$ at 6 months (Fig 6). The changes in functional $(p=0.11$ at 3 months and $p=0.18$ at 6 months) and social ( $p=0.28$ at 3 months and $p=0.46$ at 6 months) disability were not statistically significant.

\section{Discussion}

This prospective study aimed to evaluate whether LTM could improve different aspects of the oral phase of swallowing (lip continence, mastication, and disability) in patients with FP. We found that the technique of LTM significantly improves lip force and reduces drooling at 6 months after surgery and thus reduces physical disability in FP patients.

Preoperatively, patients in this study presented swallowing problems. The main complaint was drooling due to labial incompetence on the side of the palsy. The majority of patients $(n=9)$ had a score of $>4$ on the DSFS, indicating frequent episodes of moderate drooling. Oral bolus residue after swallowing was increased because of failure of the buccinator muscle to play its role of pushing food back from the vestibule. 12 patients continuously presented a bolus residue. 
At 3 months after surgery 10 patients showed increase in lip force and 2 of them showed decrease in lip force. Drooling improved in 5 patients, was unchanged in another 5 off, and was worse in 2 off.

In one patient, the worsening of symptoms was due to a scarring retraction of the nasolabial fold, which aggravated the lip incontinence. Bolus residue decreased in 8 patients and was unchanged in 4 off; no patient had increase in bolus residue. Disability perception was decreased in most of the patients $(n=9)$. At 3 months after surgery, re-education of the temporalis muscle, which is a masticatory muscle, was still in its early stage and patients had not yet developed a spontaneous smile.

At 6 months after surgery the results were more satisfactory. Re-education was continuing, and patients had developed spontaneous smiles. Lip force was improved in 12 patients. The mean lip force value was $91.15 \mathrm{mmHg}$, i.e., a $36 \%$ increase over the preoperative value. The patient with the scarring complication was the only one who did not show improvement in lip force. Drooling was decreased in 7 patients and unchanged in 5 off; however, the latter group admitted that the frequency of drooling incidents was reduced. The DSFS score was $\leq 4$ in 11 patients (mild drooling). Bolus residue after mastication also showed marked decrease in 11 patients; 8 patients had a score of 0 . Disability was decreased in 9 patients, with 5 having a score of 0 . Only the patient with scarring complication continued to have moderate handicap. The mean total score was 8.69 , i.e., a fall of $30 \%$ from baseline. The mean physical handicap score decreased by $44 \%$; the scores for functional and social handicap did not show much change. 
The discrepant results compared to series for some patients can be explained by the age (80 years old). Hembd et al. (16) reported that LTM is not as successful for restoration of smile in patients $>70$ years of age as it is in younger patients. It is possible that the effect of LTM on swallowing follows a similar pattern. Moreover, the benefits of this surgery is closely linked to proper re-education, and all patients may not show the same dedication.

Our findings are consistent with earlier reports. Byrne et al. (17) used a different surgical technique-transfer of the temporal muscle tendon to the lip commissureand reported satisfactory improvement in swallowing in FP patients. The authors also reported improvement in food satisfaction (as assessed with a self-administered questionnaire). Choi et al. (3) used electrical stimulation of facial muscles and reported improvement in swallowing efficiency.

Starmer et al. (5) showed that FP reduces lip force by about $69 \%$, leading to bolus leakage. He also demonstrated that hyaluronic acid injections to restore volume increased lip force on the palsy side by about 1.4 times, but it was still inferior to the healthy side. This is consistent with our study in which LTM increased lip force by a mean of 1.55 times. Panciera et al. (18) assessed disability using the Facial Disability Index (FDI) and demonstrated significant improvement in disability, with the FDI score increasing from 33.4 points to 49.9 points i.e. an improvement of $33 \%$ $(p<0.001)$. These results are concordant with our study, in which the DHI score changes falls from 12.3 points to 8.69 points, i.e., a reduction of $30 \%$. 
Laure et al. (19) reported improvement in dysarthria in FP patients after LTM, proving that the technique has impact on multiple aspects of FP, including esthetics, phonation, and feeding. Rozen et al. (20) used an alternative technique-gracilis free neuromuscular flap innervated by the masseteric nerve—but found that many patients complain of involuntary contractions during mastication. Therefore, for all these reasons and the fact that the LTM according to Labbé presents a reduced operating time and less comorbidities, we use preferably this technique in our Department instead of the gracilis neurotized technique. Moreover, other positive points are a similar efficiency and reliability with no risk of failure related to microvascular anastomosis $(21,22)$.

This study has been carried out on a short-term period (10 months) with a limited number of patients enrolled due to the specificity of this surgery. As such, this is a limitation. Another limitation is the use of self-administered questionnaires including a part of subjectivity in the answers. The visual assessment of the bolus residue is also a potential bias.

\section{Conclusion}

LTM followed by orofacial reeducation may reduce the dysfunction in the oral phase of swallowing. The findings of this study need to be confirmed in larger prospective studies with long-term follow-up. 


\section{CONFLICT OF INTEREST STATEMENT}

None

\section{REFERENCES}

1. Alvarez V, Dussoix P, Gaspoz J-M. [Facial palsy: diagnosis and management by primary care physicians]. Rev Med Suisse. 2009 Jan 28;5(188):258-62.

2. Ghali S, MacQuillan A, Grobbelaar AO. Reanimation of the middle and lower face in facial paralysis: review of the literature and personal approach. J Plast Reconstr Aesthetic Surg JPRAS. 2011 Apr;64(4):423-31.

3. Choi J-B. Effect of neuromuscular electrical stimulation on facial muscle strength and oral function in stroke patients with facial palsy. J Phys Ther Sci. 2016 Sep;28(9):2541-3.

4. Ertekin C, Eryaşar G, Gürgör N, Arıcı S, Secil Y, Kurt T. Orbicularis oculi muscle activation during swallowing in humans. Exp Brain Res. 2013 Jan;224(1):79-91.

5. Starmer H, Lyford-Pike S, Ishii LE, Byrne PA, Boahene KD. Quantifying Labial Strength and Function in Facial Paralysis: Effect of Targeted Lip Injection Augmentation. JAMA Facial Plast Surg. 2015 Aug;17(4):274-8.

6. de Swart BJM, Verheij JCGE, Beurskens CHG. Problems with eating and drinking in patients with unilateral peripheral facial paralysis. Dysphagia. 2003;18(4):26773.

7. Seçil Y, Aydogdu I, Ertekin C. Peripheral facial palsy and dysfunction of the oropharynx. J Neurol Neurosurg Psychiatry. 2002 Mar;72(3):391-3. 
8. Movérare T, Lohmander A, Hultcrantz M, Sjögreen L. Peripheral facial palsy: Speech, communication and oral motor function. Eur Ann Otorhinolaryngol Head Neck Dis. 2016 Nov 8;

9. Labbé D. [Lengthening of temporalis myoplasty and reanimation of lips. Technical notes]. Ann Chir Plast Esthet. 1997 Feb;42(1):44-7.

10. Chan JYK, Byrne PJ. Management of facial paralysis in the 21st century. Facial Plast Surg FPS. 2011 Aug;27(4):346-57.

11. Labbé D. [Lengthening temporalis myoplasty]. Rev Stomatol Chir Maxillofac. 2002 Apr;103(2):79-83.

12. Sforza C, Frigerio A, Mapelli A, Tarabbia F, Annoni I, Colombo V, et al. Double-powered free gracilis muscle transfer for smile reanimation: A longitudinal optoelectronic study. J Plast Reconstr Aesthetic Surg JPRAS. 2015 Jul;68(7):930-9.

13. Nduka C, Hallam M-J, Labbe D. Refinements in smile reanimation: 10-year experience with the lengthening Temporalis Myoplasty. J Plast Reconstr Aesthetic Surg JPRAS. 2012 Jul;65(7):851-6.

14. Sjögreen L, Lohmander A, Kiliaridis S. Exploring quantitative methods for evaluation of lip function. J Oral Rehabil. 2011 Jun;38(6):410-22.

15. Srivanitchapoom P, Pandey S, Hallett M. Drooling in Parkinson's disease: a review. Parkinsonism Relat Disord. 2014 Nov;20(11):1109-18. 
16. Hembd A, Harrison B, Rocha CSM, Rocha F de S, Chamseddin K, Labbé D, et al. Facial Reanimation in the Seventh and Eighth Decades of Life: Plast Reconstr Surg. 2018 May;141(5):1239-51.

17. Byrne PJ, Kim M, Boahene K, Millar J, Moe K. Temporalis tendon transfer as part of a comprehensive approach to facial reanimation. Arch Facial Plast Surg. 2007 Aug;9(4):234-41.

18. Panciera DT, Sampieri C, Deganello A, Danesi G. Lengthening Temporalis Myoplasty: Objective Outcomes and Site-Specific Quality-of-Life Assessment. Otolaryngol--Head Neck Surg Off J Am Acad Otolaryngol-Head Neck Surg. 2017 Jun $1 ; 194599817717458$.

19. Laure B, Fritz A-H, Dufour J, Goga D. [Treatment of facial paralysis with temporalis lengthening myoplasty and dysarthria improvement]. Ann Chir Plast Esthet. 2013 Apr;58(2):96-102.

20. Rozen S, Harrison B. Involuntary movement during mastication in patients with long-term facial paralysis reanimated with a partial gracilis free neuromuscular flap innervated by the masseteric nerve. Plast Reconstr Surg. 2013 Jul;132(1):110e-6e.

21. Veyssière A, Labbé $\mathrm{D}$, Bénateau $\mathrm{H}$. Lengthening temporalis myoplasty and facial paralysis from birth. J Plast Reconstr Aesthetic Surg JPRAS. 2015 Mar;68(3):312-20.

22. Bos R, Reddy SG, Mommaerts MY. Lengthening temporalis myoplasty versus free muscle transfer with the gracilis flap for long-standing facial paralysis: A 
systematic review of outcomes. J Cranio-Maxillo-fac Surg Off Publ Eur Assoc

Cranio-Maxillo-fac Surg. 2016 Aug;44(8):940-51.

\section{Legends}

Table 1:

Cohort characteristics

Fig 1:

A. Nine years old patient with a right congenital facial palsy. We can notice the asymmetric smile.

B. At 6 months postoperative the smile is spontaneous and symmetric.

Fig 2:

Photography of the technique used to measure the lip force.

Fig 3: Lip force results

Average with confidence interval of $95 \%$ and minimal and maximal value at the 3 checkpoints of the lip force. The average rises up significantly between preoperative and 6 months postoperative $\left({ }^{*} p<0,05\right)$

Fig 4: DSFS results

Average with confidence interval of $95 \%$ and minimal and maximal value at the 3 checkpoints of the DSFS results. The average decreases significantly between preoperative and 6 months postoperative $\left({ }^{*} p<0,05\right)$ 
Fig 5: Bolus residue results

Average with confidence interval of $95 \%$ and minimal and maximal value at the 3 checkpoints of the Bolus residue results. The average decreases significantly between preoperative and 6 months postoperative $\left({ }^{*} p<0,05\right)$

Fig 6: DHI: subcategory physical results Average with confidence interval of $95 \%$ and minimal and maximal value at the 3 checkpoints of the DHI results (subcategory physical). The average decreases significantly between preoperative and 6 months postoperative $\left({ }^{*} p<0,05\right)$ 


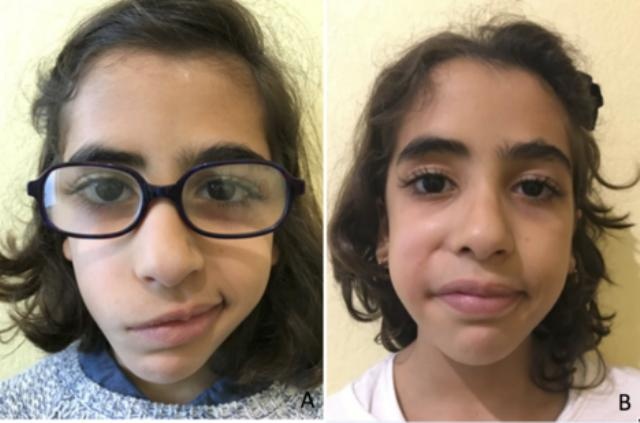




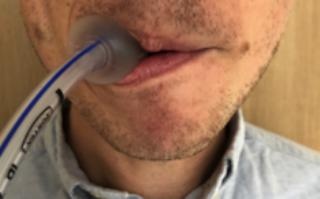


Lip force

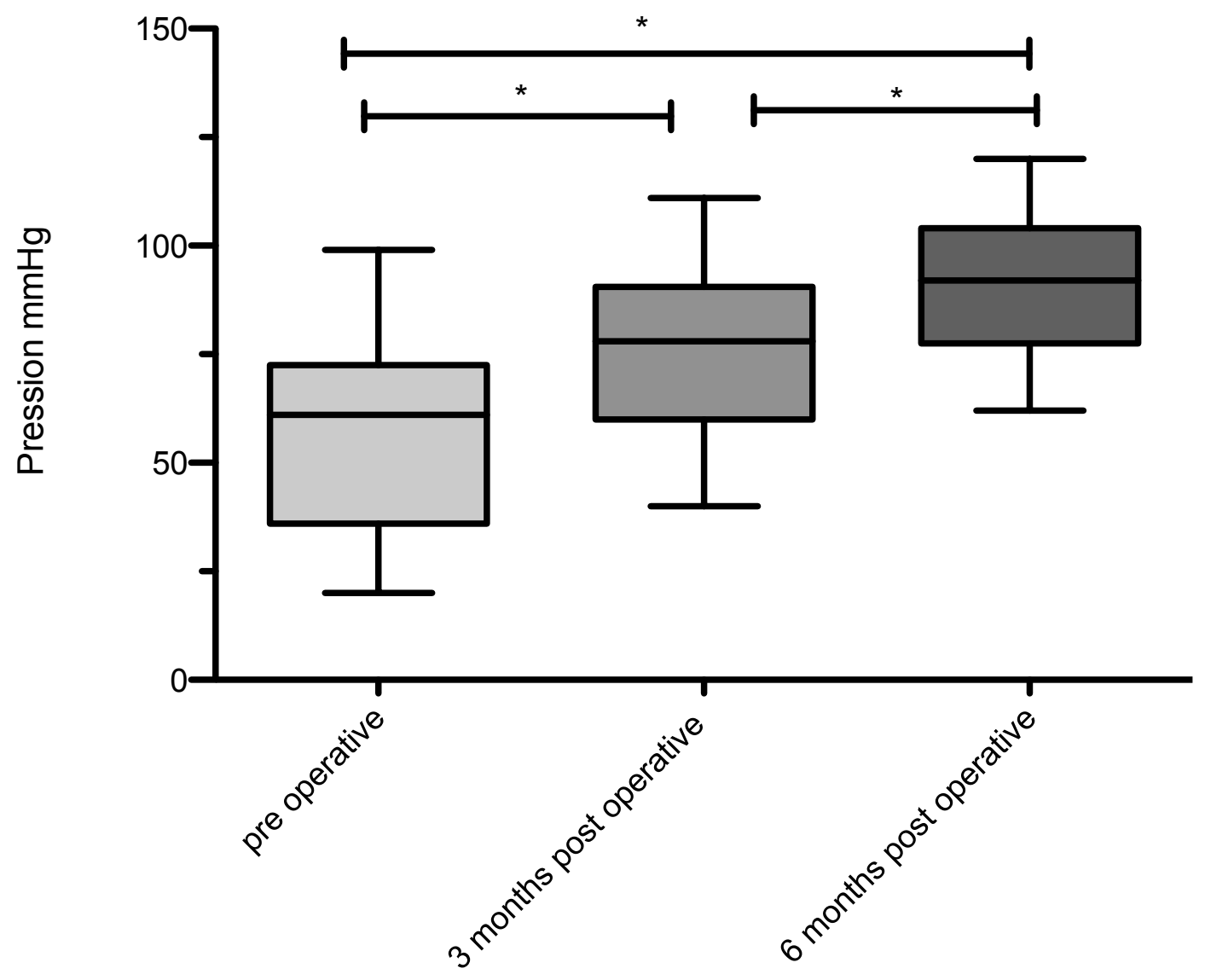

Time 
DSFS

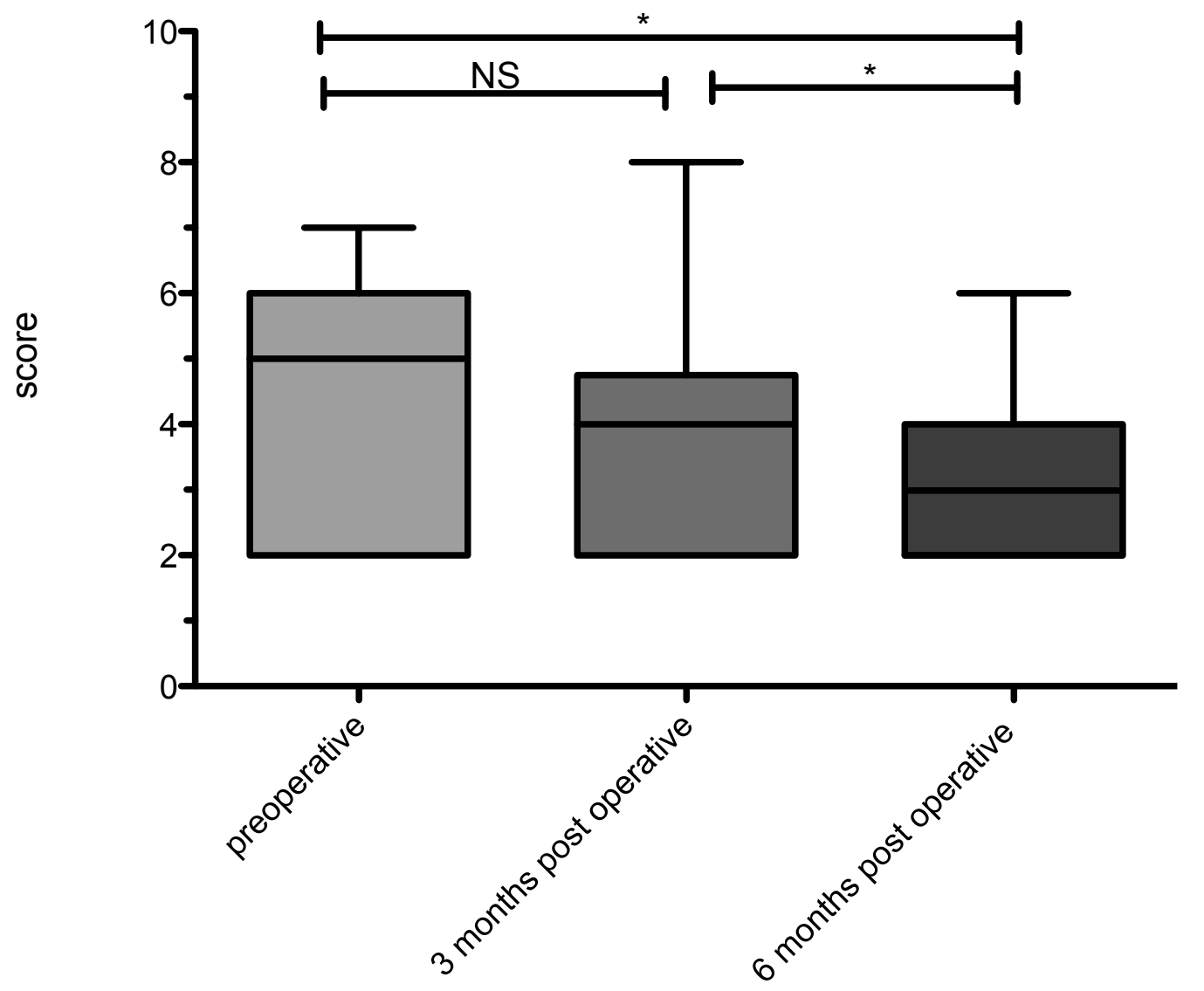

Time 


\section{Bolus residue}

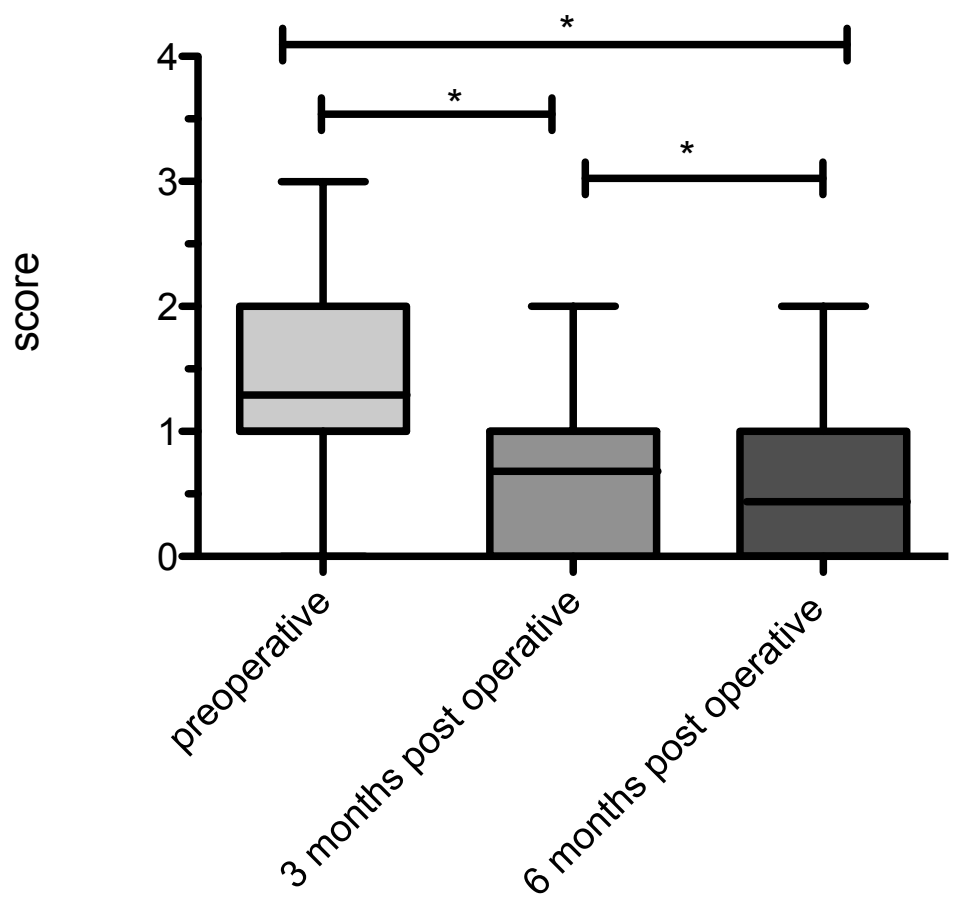

Time 
$\mathrm{DHI}$ : subcategory physical

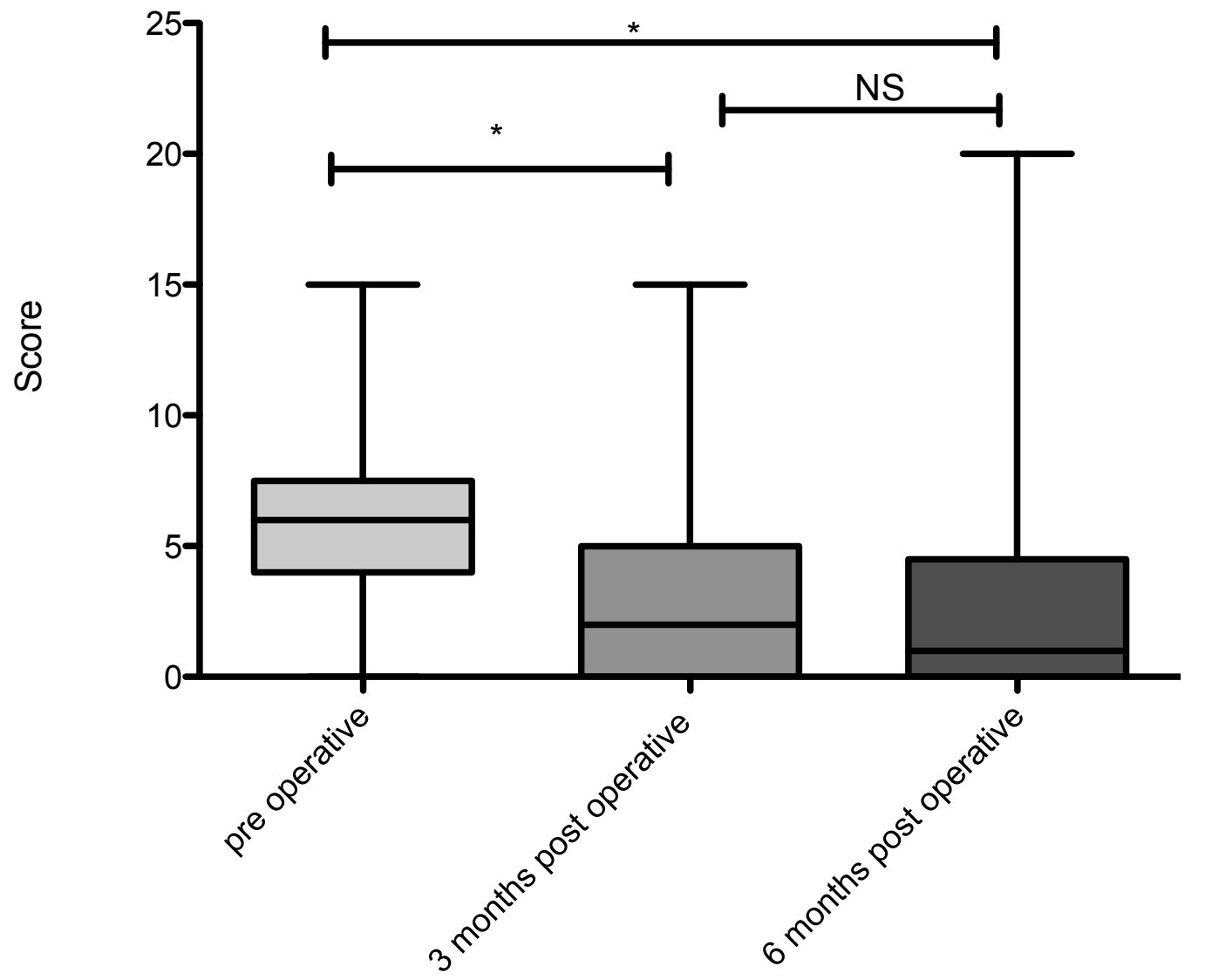

Time 


\begin{tabular}{|r|l|r|l|r|}
\hline Patient & Sexe & Age (y) & Etiology & Duration of palsy (months) \\
\hline 1 & M & 56 & Right acoustic neurinoma & 23 \\
\hline 2 & M & 9 & Congenital facial Palsy & 108 \\
\hline 3 & F & 66 & Right acoustic neurinoma & 20 \\
\hline 4 & F & 10 & Congenital facial Palsy & 120 \\
\hline 5 & M & 10 & Congenital facial Palsy & 120 \\
\hline 6 & M & 72 & Right acoustic neurinoma & 144 \\
\hline 7 & F & 58 & Left Facial palsy zona & 19 \\
\hline 8 & F & 47 & Left Facial palsy of Charles Bell & 60 \\
\hline 9 & M & 80 & Left parotidectomy & 18 \\
\hline 10 & F & 64 & Left acoustic neurinoma & 11 \\
\hline 11 & F & 59 & Left cerebral meningioma & 19 \\
\hline & & & Right cerebral cavernoma & 23 \\
\hline 12 & F & 54 & hemorrhage & 18 \\
\hline 13 & M & 53 & Right parotidectomy & \\
\hline & & & & \\
\hline
\end{tabular}

